\title{
Simultaneous Video Assisted Thoracoscopic Costal and Femoral Osteochondromas Resection in a Child with Multiple Exostoses Disease: A Case Report and Literature Review
}

\author{
Cosimo Gigante ${ }^{1 *}$, Giacomo Maso ${ }^{2}$, Giovanni Baldin ${ }^{2}$ and Piergiorgio Gamba ${ }^{3}$ \\ ${ }^{1}$ Pediatric Orthopaedic Unit, Italy \\ ${ }^{2}$ Department of Orthopedics and Orthopedic Oncology, Italy \\ ${ }^{3}$ Pediatric Surgery Unit University of Padova, Italy
}

*Corresponding author: Cosimo Gigante, Pediatric Orthopaedic Unit, Azienda Ospedaliera Padova, via N Giustiniani 2; 35128

Padova, Italy

\section{ARTICLE INFO}

Received: 峵 August 11, 2019

Published: 幽 August 19, 2019

Citation: Cosimo Gigante, Giacomo Maso, Giovanni Baldin, Piergiorgio Gamba. Simultaneous Video Assisted Thoracoscopic Costal and Femoral Osteochondromas Resection in a Child with Multiple Exostoses Disease: A Case Report and Literature Review. Biomed J Sci \& Tech Res 20(4)-2019. BJSTR. MS.ID.003484.

\section{ABSTRACT}

The case reported concerns a 5-years-old child affected by Hereditary Multiple Exostoses (HME), a genetic disorder with autosomal dominant inheritance, that cause multiple skeletal exostosis. Usually those masses are asymptomatic, but in some cases, they can cause a huge spectrum of problems due to their size and site localization interfering with surrounding structures such as nerves, vessels, or organs. In order to reduce patient distress and hospital stay we resected in the same surgical session, an exostosis on the left knee (that was interfering with the correct bone growth) and, with video-assisted-thoracoscopy (VATS), three rib exostosis (one of them misdiagnosed at the MRI examination).. Temporary femoral hemiepiphysiodesis was performed too in order to correct the knee valgus malalignment. In case of exostosis localized the internal side of the ribs, the collaboration of a skilled pediatric surgeon is recommended to perform VATS resection.

\section{Introduction}

Osteochondroma, also called exostosis, is the most common cartilaginous tumour, which is estimated to account for 20 to $25 \%$ of all benign bone tumours. The most frequent localization is in the long bones: distal femur, proximal humerus and proximal tibia. It originates from the metaphysis but, with skeletal growth, it tends to move toward the diaphysis. Only about $3 \%$ of osteochondromas occur in vertebrae and ribs; among them, $70 \%$ are accompanied by hereditary multiple exostoses (HME) [1]. Solitary osteochondromas occur sporadically and arise from a defect in the perichondral ring (en coach of Ranvier) or might be secondary to a traumatic insult. The exostosis (i.e.) are multiple in the hereditary multiple exostoses (HME). HME is a genetic disorder with an autosomal dominant inheritance and a variable penetrance involving EXT gene family. Most common mutations involve gene EXT1 on chromosome 8 and gene EXT2 on chromosome 11. Multiple skeletal lesions are usually diffused and relatively symmetrical and typically, osteochondromas involve the bone circumferentially, mostly surrounding the metaphyseal regions, causing swelling and sometimes limiting joint motion. In severe forms, limb shortening, and deformity are associated.

The prevalence of HME in European populations is about 1 case in 50,000 people. The exostoses usually present as painless masses, however sometimes they can cause problems due to interference from surrounding anatomical structures, such as reactive tenosynovitis, nervous or vascular compression, and if located in the ribs even haemothorax or pneumothorax caused by damage to the pleura. Malignant transformation in chondrosarcoma is more common in the setting of multiple hereditary osteochondromato- 
sis (about 4\% risk) [2]. We reported a 5 years old child affected by HME presenting three costal osteochondroma successfully treated by video-assisted thoracoscopic surgery (VATS). In the same surgical session was also removed a large femoral exostosis associated to a routinely emiepiphysiodesys in order to correct secondary valgus of the knee.

\section{Case Presentation}

A 5 years old child, affected by HME, came to our observation for two great exostoses respectively localized at the $2 \mathrm{nd}$ rib of left hemithorax and at the left distal femur (where a secondary valgus knee was quickly worsening) (Figures 1a-1d). The patient, asymptomatic and free from other illnesses, was candidate for surgery because of the size and the potential risk of malignant transformation of the costal and femoral exostoses and for the correction of the valgus deformity of the knee. The removal of the femoral exostosis was decided also to prevent future major surgery conditioned by the close relationships of the exostosis with the regional great blood vessels.MR Angiography of the lower limbs and thorax MR were performed preoperatively (Figures 1e-1g); these studies showed three major exostosis: one of at the left femur (25x25 mm and an extension of $37 \mathrm{~mm}$ ), one at the external side of the $2^{\text {nd }}$ left rib(37x $21 \mathrm{~mm}$ and an extension of $35 \mathrm{~mm}$ ) and an unexpected one at the internal side of the $5^{\text {th }}$ right rib $(23 \times 14 \mathrm{~mm}$ and an extension of $30 \mathrm{~mm}$ ).

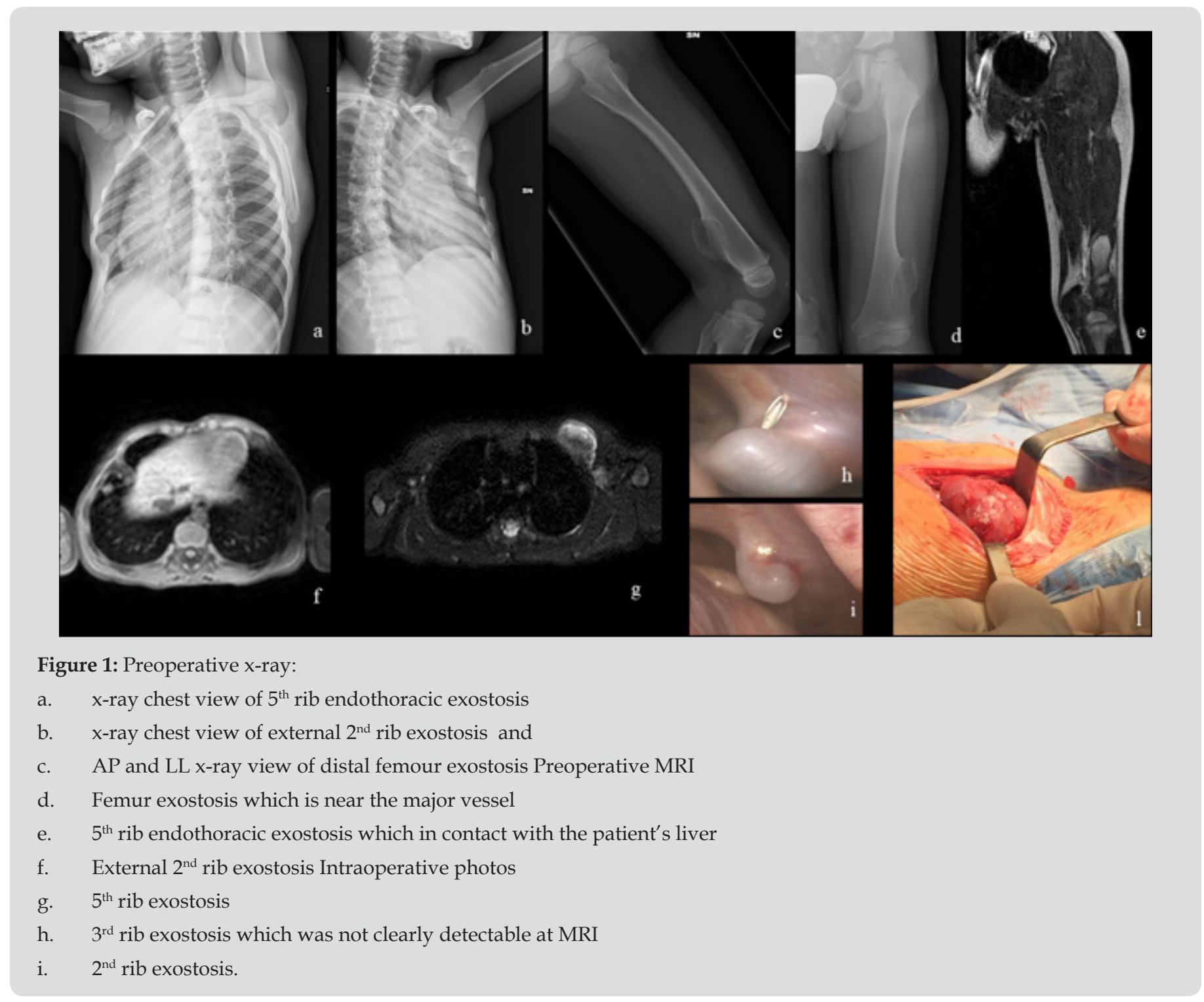

As this last exostosis was pushing on the diaphragm imprinting the liver, it was also decided to remove it, in a one-step surgery with the others, performing a video assisted thoracoscopic costal resection.To improve post-operative pain control the patient was firstly prepared for surgery with ultrasound guided femoral block nerve and secondly with general anaesthesia. The child was placed on the operating table in supine decubitus with both the lower limbs included in the sterile field in order to allow a vascular bypass in case of accidental intraoperative damaging of the femoral artery. At first the lateral femoral exostosis was isolated and then resected 
through a large lateral surgical approach. Subsequently, through a distinct little medial access, a routinely medial emi-epyphisiodesis of the distal femur was performed using Orthofix®'sEight-Plate $®$.

At the end of the femoral procedure the patient was positioned on left side decubitus to allow the general pediatric surgeon to perform Video-Assisted Thoracoscopic Surgery (VATS). Through a $5 \mathrm{~mm}$ trocar introduced close the lower margin of the right chest, after insufflation, the osteochondroma localized at the inner side of the right 5 th was identified (Figure $1 \mathrm{~h}$ ). At this level the rib was exposed by an open access and then resected en bloc with the exostosis, checking with the thoracoscopy for unintentional pleural damage.

During the procedure we found another misdiagnosed endothoracic osteochondroma, located at the internal side of the 3rd right rib (Figure 1i), that was removed in the same way. Then, we checked with thoracoscopy for abnormal bleeding, without finding any. Through the thoracoscopy entry point, we placed a thorax drain of $10 \mathrm{Fr}$ fixed with a silk knot. Then we proceeded to sew up the wound with absorbable material. In the last surgical time, with the patient in supine decubitus, we approached in open way to the last exostosis designed to be removed, which was in the outer part of the second left rib (Figure 1j). At first, we isolated the exostosis with blunt tools spreading apart the pectoralis major and the pectoralis minor and then resected it with the chisel. The wound was then sewed up and we placed a bandage. All the removed exostosis were sent to the Pathological Anatomy ward, and they all were later confirmed to be osteochondromas. After the surgery the patient had no complications and was dismissed from the pediatric ward 2 days after surgery. At the follow-up visits, the patient never complained to suffer for pain or presented any other problem. Right now, he's fine and he's still followed with periodical visits to check his growth development and progressive correction of the valgus deformity of the knee.

\section{Discussion}

Osteochondromas are the most common benign bone neoplasms. They occur in 3\% of the general population and account for more than $30 \%$ of all benign bone tumours and for $10 \%-15 \%$ of all bone tumours. Exostosis can occur as a sporadic solitary lesion with no hereditary link in $85 \%$ of cases, otherwise may be multiple in the context of hereditary multiple exostoses (HME) [3,4]. HME is a rare autosomal-dominant paediatric musculoskeletal disorder with an incidence of about 1:50000 characterized by significant number of osteochondromas that form next to the growth plates of many skeletal elements, including long bones, ribs and vertebrae [5]. HME cases are associated with heterozygous loss-of-function mutations in EXT1 and EXT 2 genes which are involved in heparan sulfate synthesis.

Although in most cases exostoses are asymptomatic, in some children they may grow quickly reaching remarkable dimensions and causing mechanical pressure on the adjacent anatomical structures. Moreover, because of their interference with the growing activity of the physis, they may cause growth retardation, deformities and impingement of surrounding anatomical elements (including other bones, spinal cord, muscles, tendons, blood vessels and nerves).For these relevant functional and cosmetic problems surgical removal is recommended in selected cases and localizations [5]. Moreover it should not be underestimated that, in patients with HME, there is the possibility of malignant degeneration to chondrosarcoma (reported to be about 4\%) [2] or, more rarely, other malignancies that can be life threatening because of their resistance to chemo-therapy or radiation therapy.

Apart malignant degeneration, chest complications of thoracic osteochondromas are rare, but cases of haemothorax and pneumothorax consequent to costal exostosis with HME have been reported in literature [6,7]. The aetiology of the damage is either the shearing of pleura or diaphragm by the intrathoracic extrusion of costal exostosis, or focal pleural changes induced by the friction and subsequent inflammation between the pleura and the osteochondroma [8]. To our knowledge, this is the first case reported in English literature of multiple exostoses removed both from the chest and other extremities on the same surgical session. This decision was made to reduce as much as possible the distress for the patient and the post-surgery days in hospital.

To achieve such an impressive result VATS was necessary to remove the rib exostoses. The thorax MR helped in both establishing the presence and size of the exostoses and in planning the surgical treatment while with direct thoracoscopic vision of the mass we were able to perform surgery in the best conditions reducing potential life-treating damage. Traditionally, patients who had a costal exostosis had undergone thoracotomy to remove the lesion and prevent complications. However, VATS have been increasingly used as the main way to treat these kinds of exostoses. Compared with a thoracotomy, VATS allow for less invasive approach to resection, with fewer post-surgery complications, pain, and better outcome both cosmetically and in terms of recovery [9-13].

Although costal exostoses in children and adolescents are usually asymptomatic, they can rarely lead to serious complications due to their size and interference with other structures (such as nerves and vessels). In the absence of symptoms, there is no clear indication for surgical treatment, but many authors have suggested to treat inward-protruding osteochondromas [14,15]. It's generally agreed to avoid any treatment or postpone the operation until adolescence in HME patients if there are no symptoms. Our decision to proceed with thoracic surgery, even if the patient had no thoracic symptoms, was due to the young age of the patient, with the consequent high probability that both the exostoses could increase in size in the future (being the left external exostosis already very large now).Moreover, if the removal of the right rib-exostoses was largely justified to avoid future complications because of its 
position close to the liver and to the diaphragm [9], the removal of the external 2nd left rib exostosis seemed appropriate because its already considerable size. Also, even if it's relatively rare, malignant transformation to chondrosarcoma is possible (reported in about 4 $\%$ of patients with HME) and in our opinion is best to reduce that possibility in such a complex site [2].

In addition, in our 5-year-old patient, the left distal femur osteochondroma was interfering with the growth and with the alignment of the left lower limb so it was necessary to perform the exostosis excision and the emi-epiphysiodesis. Moreover, the benign tumour was close to the femoral artery and by removing it our proposal was to avoid the risk of future vessels compression and potential secondary pseudo-aneurysm due to growth of the exostosis [16,17]. Temporary emi-epiphysiodesis is a wellknown surgical procedure able to correct valgus knee in skeletally immature patients and in particular it isn't the main topic of our case presentation. In literature patients with HME frequently have a high rate of knee deformity, mainly a genu valgum deformity [18]

Few studies reported the results of emi-epiphysiodesis in patients with HME and correlated genuvalgus deformity, but according to Kang et al. who analysed the results of $15 \mathrm{HME}$ patients it is a validated surgical procedure. Kang et al. compared the outcomes of HME patients with genu valgum after temporary emiepiphysiodesis to those of patients with idiopathic cause for knee deformity. The satisfactory correction was comparable between the two groups, but it is interesting to underline that HME patients showed lower correction velocity and required a longer time by about six months for correction, compared with the idiopathic knee deformity group. The exostoses can influence longitudinal limb growth and the abnormal growth capacity of physes around the knee due to abnormal chondrocytes function is thought to be the main cause for slow correction velocity $[18,19]$.

So, the authors decided to consider the temporary emi-epiphysiodesis at the early age of the patient. At last before performing a surgery in HME patients a MRI with contrast enhancementis an important imaging technique that can provide useful information for many reasons: first to better understand the benign or malignant nature of the lesions MRI (i.e. for visualization of the cartilage cap covering the exostosis) and secondly to view soft tissue and anatomical structures (such as vessels, nerve, thorax, etc.) around the osteochondroma that a surgeons have to consider and preserve [20].

\section{Conclusion}

Even if the treatment of young patients affected with HME is not mandatory, is still worth to take into consideration the possibility to perform preventive surgery if the masses are of considerable size and/or in dangerous positions (near vessels, nerves or internal organs). In our experience in case of multiple localizations performing surgery in one stage session should be preferred to reduce the patient distress and hospital stay.
Pre-operatory thoracic MR can play a necessary role in studying potential malignant lesion and in the surgery-planning in case of ribs exostoses, helping to determine their position, size, connection with their surroundings, and most importantly showing undetected masses. Moreover, to assure the best outcome, it's strongly recommended a multidisciplinary approach performing the excision of the exostosis localized at the internal side of the ribs using VATSby the help and collaboration of the pediatric surgeon; this can help to prevent complications such as accidental lesions, and minimize intra-operatory risks linked to thoracic surgery.

\section{Acknowledgement}

None.

\section{Conflict of Interest}

No conflict of interest.

\section{Reference}

1. Masoum SHF, Moradi A, Ebrahimzadeh MH (2014) Multiple rib exostoses in a boy: a rare case resulting in surgery secondary to cosmetic concerns. Arch bone Jt Surg 2(3): 243-245.

2. Fei L, Ngoh C, Porter DE (2018) Chondrosarcoma transformation in hereditary multiple exostoses: A systematic review and clinical and costeffectiveness of a proposed screening model. J Bone Oncol 13: 114-122.

3. Kitsoulis P, Galani V, Stefanaki K, Paraskevas G, Karatzias G, et al. (2008) Osteochondromas: review of the clinical, radiological and pathological features. In Vivo 22(5): 633-646.

4. Kikuchi R, Mino N, Matsukura T, Hirai T (2010) Resected osteochondroma of the rib in an elderly patient. Gen Thorac Cardiovasc Surg 58(11): 588591.

5. Pacifici M (2017) Hereditary Multiple Exostoses: New Insights into Pathogenesis, Clinical Complications, and Potential Treatments. Curr Osteoporos Rep 15(3): 142-152.

6. Takata K, Suzuki K, Kurosaki Y (2008) Spontaneous hemothorax in hereditary multiple exostosis involving the ribs. Radiat Med 26(1): 3941.

7. Cowles RA, Rowe DH, Arkovitz MS (2005) Hereditary multiple exostoses of the ribs: an unusual cause of hemothorax and pericardial effusion. J Pediatr Surg 40(7): 1197-1200.

8. Huang L, Lu Q, Yan X, Han Y (2014) A 5-year-old boy with a large hereditary multiple exostoses lump grown into thoracic cavity. Ann Thorac Cardiovasc Surg 20: 528-530.

9. Assefa D, Murphy RC, Bergman K, Atlas AB (2011) Three faces of costal exostoses: case series and review of literature. Pediatr Emerg Care 27(12): 1188-1191.

10. Nakano T, Endo S, Tsubochi H, Tetsuka K (2012) Thoracoscopic findings of an asymptomatic solitary costal exostosis: is surgical intervention required? Interact Cardiovasc Thorac Surg 15(5): 933-934.

11. Khosla A, Parry RL (2010) Costal osteochondroma causing pneumothorax in an adolescent: a case report and review of the literature. J Pediatr Surg 45(11): 2250-2253.

12. Patel M, Bauer TW, Santoscoy T, Ilaslan H (2015) Osteochondroma of the fifth rib resulting in recurrent hemothorax. Skeletal Radiol 44(12): 1853-1856.

13. Dumazet A, Launois C, Dury S, Sailhan F, Alifano M, et al. (2018) Hereditary multiple exostoses of the ribs as an uncommon cause of pneumothorax: A case report. Medicine (Baltimore) 97(35): e11894.

14. Reynolds JR, Morgan E (1990) Haemothorax caused by a solitary costal exostosis. Thorax 45(1): 68-69. 
15. Teijeira FJ, Baril C, Younge D (1989) Spontaneous hemothorax in a patient with hereditary multiple exostoses. Ann Thorac Surg 48(5): 717718.

16. Belmir H, Azghari A, Mechchat A, Benzirar A, Idrissi R, et al. (2011) [Rupture of a popliteal artery pseudo-aneurysm revealing a tibia osteochondroma: case report and review of the literature]. J Mal Vasc 36(1): 50-55

17. Sakata T, Mogi K, Sakurai M, Nomura A, Fujii M, et al. (2017) Popliteal Artery Pseudoaneurysm Caused by Osteochondroma. Ann Vasc Surg 43 313.e5-313.e7.

\section{ISSN: 2574-1241}

DOI: 10.26717/BJSTR.2019.20.003484

Cosimo Gigante. Biomed J Sci \& Tech Res

(C) This work is licensed under Creative

Submission Link: https://biomedres.us/submit-manuscript.php
18. Kang S, Kim JY, Park SS (2017) Outcomes of Hemiepiphyseal Stapling for Genu Valgum Deformities in Patients with Multiple Hereditary Exostoses: A Comparative Study of Patients with Deformities of Idiopathic Cause. J Pediatr Orthop 37(4): 265-271

19. Jones KB (2011) Glycobiology and the growth plate: current concepts in multiple hereditary exostoses. J Pediatr Orthop 31(5): 577-586.

20. De Souza AMG, Bispo Júnior RZ (2014) Osteochondroma: ignore or investigate? Rev Bras Ortop 49(6): 555-564.

\begin{tabular}{ll} 
BIOMEDICAL & Assets of Publishing with us \\
\hline RESEARCHES & - Global archiving of articles \\
\hline & - Immediate, unrestricted online access \\
\hline
\end{tabular}

